\title{
An Ordered Lasso and Sparse Time-lagged Regression
}

\author{
Xiaotong Suo* Robert Tibshirani ${ }^{\dagger}$ \\ Institute for Computational \& Mathematical Engineering, \\ and Departments of Health Research \& Policy, and Statistics, Stanford \\ University
}

\begin{abstract}
We consider a regression scenario where it is natural to impose an order constraint on the coefficients. We propose an order-constrained version of $\ell_{1}$-regularized regression (lasso) for this problem, and show how to solve it efficiently using the well-known Pool Adjacent Violators Algorithm as its proximal operator. The main application of this idea is to time-lagged regression, where we predict an outcome at time $t$ from features at the previous $K$ time points. In this setting it is natural to assume that the coefficients decay as we move farther away from $t$, and hence the order constraint is reasonable. Potential application areas include financial time series and prediction of dynamic patient outcomes based on clinical measurements. We illustrate this idea on real and simulated data.
\end{abstract}

\section{Introduction}

Suppose that we observe data $\left(\mathbf{x}_{i}, y_{i}\right)$ for $i=1,2, \ldots, N$ where $N$ is the number of observations, $\mathbf{x}_{i}=\left(x_{i 1}, x_{i 2}, \ldots, x_{i p}\right)$ is a vector of $p$ feature measurements, and $y_{i}$ is a response value. We consider the usual linear regression

\footnotetext{
*email:xiaotong@stanford.edu

$\dagger$ †email:tibs@stanford.edu, Supported by NSF Grant DMS-99-71405 and National Institutes of Health Contract N01-HV-28183
} 
framework

$$
y_{i}=\beta_{0}+\sum_{j=1}^{p} x_{i j} \beta_{j}+\epsilon_{i}
$$

with $\mathrm{E}\left(\epsilon_{i}\right)=0$ and $\operatorname{Var}\left(\epsilon_{i}\right)=\sigma^{2}$. The lasso or $\ell_{1}$-regularized regression (Tibshirani 1996) chooses the parameters $\beta_{0}, \boldsymbol{\beta}=\left(\beta_{1}, \beta_{2}, \ldots \beta_{p}\right)$ to solve

$$
\operatorname{minimize}\left\{\frac{1}{2} \sum_{i=1}^{N}\left(y_{i}-\beta_{0}-\sum_{j=1}^{p} x_{i j} \beta_{j}\right)^{2}+\lambda \sum_{j=1}^{p}\left|\beta_{j}\right|\right\}
$$

where $\lambda \geq 0$ is a fixed tuning parameter. This problem is convex and yields sparse solutions for sufficiently large values of $\lambda$.

In this paper we add an additional order constraint on the coefficients, and we call the resulting procedure the ordered lasso. We derive an efficient algorithm for solving the resulting problem. The main application of this idea is to time-lagged regression, where we predict an outcome at time $t$ from features at the previous $K$ time points. In this case, it is natural to assume that the coefficients decay as we move farther away from $t$ so that the order (monotonicity) constraint is reasonable. A key feature of our procedure is that it automatically determines the most suitable value of $K$ for each predictor, directly from the monotonicity constraint. The paper is organized as follows. Section 2 contains motivations and algorithms for solving the ordered lasso, as well as results comparing the ordered and standard lasso on simulated data. Section 3 contains the detailed algorithms for applying the ordered lasso to the time-lagged regression. We demonstrate the usage of such algorithms on real and simulated data in Sections 3.3 and 3.4. We also apply this framework to auto-regressive (AR) time series and compare its performance to both the traditional method for fitting AR model using least squares and the Akaike information criterion, and the lasso procedure for fitting AR model. Section 4 gives a definition of degrees of freedom for the ordered lasso. Section 5 generalizes the ordered lasso to the logistic regression model. Section 6 contains some discussion and directions for future work. 


\section{Lasso with an order constraint}

\subsection{The basic idea}

We consider the lasso problem with an additional monotonicity constraint, i.e.,

$$
\operatorname{minimize}\left\{\frac{1}{2} \sum_{i=1}^{N}\left(y_{i}-\beta_{0}-\sum_{j=1}^{p} x_{i j} \beta_{j}\right)^{2}+\lambda \sum_{j=1}^{p}\left|\beta_{j}\right|\right\}
$$

subject to $\left|\beta_{1}\right| \geq\left|\beta_{2}\right| \geq \ldots \geq\left|\beta_{p}\right|$. This setup makes sense in problems where some natural order exists among the features. However, this problem is not convex. Hence we modify the approach, writing each $\beta_{j}$ as $\beta_{j}=\beta_{j}^{+}-\beta_{j}^{-}$ with $\beta_{j}^{+}, \beta_{j}^{-} \geq 0$. We propose the following problem

$$
\operatorname{minimize}\left\{\frac{1}{2} \sum_{i=1}^{N}\left(y_{i}-\beta_{0}-\sum_{j=1}^{p} x_{i j}\left(\beta_{j}^{+}-\beta_{j}^{-}\right)\right)^{2}+\lambda \sum_{j=1}^{p}\left(\beta_{j}^{+}+\beta_{j}^{-}\right)\right\},
$$

subject to $\beta_{1}^{+} \geq \beta_{2}^{+} \geq \ldots \geq \beta_{p}^{+} \geq 0$ and $\beta_{1}^{-} \geq \beta_{2}^{-} \geq \ldots \geq \beta_{p}^{-} \geq 0$. The use of positive and negative components (rather than absolute values) makes this a convex problem. Its solution typically has one or both of each pair $\left(\hat{\beta}_{j}^{+}, \hat{\beta}_{j}^{-}\right)$equal to zero, in which case $\left|\hat{\beta}_{j}\right|=\hat{\beta}_{j}^{+}+\hat{\beta}_{j}^{-}$and the solutions $\left|\hat{\beta}_{j}\right|$ are monotone non-increasing in $j$. However, this need not be the case, as it is possible for both $\hat{\beta}_{j}^{+}$and $\hat{\beta}_{j}^{-}$to be positive and the $\left|\hat{\beta}_{j}\right|$ to have some non-monotonicity. In other words, the constraints strongly encourage, but don't require, that the solutions are monotone in absolute value. A similar approach was used in the interaction models of Bien, Taylor \& Tibshirani (2013). This problem can be solved by a standard quadratic programming algorithm, and this works well for small problems. For larger problems, there is an efficient first-order generalized gradient algorithm, which uses the Pool Adjacent Violators Algorithm (PAVA) for isotonic regression as its proximal operator (for example, see de Leeuw, Hornik \& Mair (2009)). We describe this in the next subsection.

\subsection{Algorithmic details}

We assume that the predictors and outcome are centered so that the intercept has the solution $\hat{\beta}_{0}=0$. For illustrative purposes, we write our data in 
matrix form. Let $\mathbf{X}$ be the $N \times p$ data matrix and $\mathbf{y}$ be the vector of length $N$ containing the response value for each observation. We first consider the following problem

$$
\operatorname{minimize}\left\{\frac{1}{2}(\mathbf{y}-\mathbf{X} \boldsymbol{\beta})^{T}(\mathbf{y}-\mathbf{X} \boldsymbol{\beta})+\lambda \sum_{j=1}^{p} \beta_{j}\right\}
$$

subject to $\beta_{1} \geq \beta_{2} \geq \ldots \geq \beta_{p} \geq 0$. We let $h(\boldsymbol{\beta})=\lambda \sum_{j=1}^{p} \beta_{j}+\mathbb{I}_{C}(\boldsymbol{\beta})$, where $\mathbb{I}$ is an indicator function and $C$ is the convex set given by $\left\{\boldsymbol{\beta} \in \mathbb{R}^{p} \mid \beta_{1} \geq\right.$ $\left.\beta_{2} \geq \ldots \geq \beta_{p} \geq 0\right\}$. We want to calculate the proximal mapping of $h(\boldsymbol{\beta})$, i.e.,

$$
\operatorname{prox}_{h}(\boldsymbol{\beta})=\operatorname{argmin}_{\mathbf{u}}\left\{\lambda \sum_{j=1}^{p} u_{j}+\mathbb{I}_{C}(\mathbf{u})+\frac{1}{2}\|\mathbf{u}-\boldsymbol{\beta}\|^{2}\right\} .
$$

There is an elegant solution to obtain this proximal mapping. We first consider solving the following problem

$$
\operatorname{minimize}\left\{\frac{1}{2} \sum_{j=1}^{n}\left(y_{i}-\theta_{i}\right)^{2}+\lambda \sum_{i=1}^{n} \theta_{i}\right\}
$$

subject to $\theta_{1} \geq \theta_{2} \geq \ldots \geq \theta_{n} \geq 0$. The solution can be obtained from an isotonic regression using the well-known Pool Adjacent Violators Algorithm (Barlow, Bartholomew, Bremner \& Brunk 1972). In particular, if $\left\{\hat{\theta}_{i}\right\}=$ $\left\{\hat{y}_{i}^{\lambda}\right\}$ is the solution to the isotonic regression of $\left\{y_{i}-\lambda\right\}$, i.e.,

$$
\left\{\hat{\theta}_{i}\right\}=\operatorname{argmin}_{\boldsymbol{\theta}}\left\{\frac{1}{2} \sum_{i=1}^{n}\left(y_{i}-\lambda-\theta_{i}\right)^{2}\right\},
$$

subject to $\theta_{1} \geq \theta_{2} \geq \ldots \geq \theta_{n}$, then $\left\{\hat{y}_{i}^{\lambda} \cdot \mathbb{I}\left(\hat{y}_{i}^{\lambda}>0\right)\right\}$ solves problem (4). Hence the solution to $(3)$ is

$$
\operatorname{prox}_{h}(\boldsymbol{\beta})=\hat{\boldsymbol{\beta}}^{\lambda} \cdot \mathbb{I}\left(\hat{\boldsymbol{\beta}}^{\lambda}>0\right) .
$$

Using this in the proximal gradient algorithm, the first-order generalized gradient update step of $\boldsymbol{\beta}$ for solving (2) is

$$
\boldsymbol{\beta} \leftarrow \operatorname{prox}_{\gamma h}\left(\boldsymbol{\beta}-\gamma \mathbf{X}^{T}(\mathbf{X} \boldsymbol{\beta}-\mathbf{y})\right)
$$


The value $\gamma>0$ is a step size that is adjusted by backtracking to ensure that the objective function is decreased at each step. To solve (1) we augment each predictor $x_{i j}$ with $x_{i j}^{*}=-x_{i j}$ and write $x_{i j} \beta_{j}=x_{i j} \beta_{j}^{+}+x_{i j}^{*} \beta_{j}^{-}$. We denote the expanded parameters by $\left(\boldsymbol{\beta}^{+}, \boldsymbol{\beta}^{-}\right)$and apply the proximal operator (7) alternatively to $\mathbf{X}$ and $\mathbf{X}^{*}$ to obtain the minimizers $\left(\hat{\boldsymbol{\beta}}^{+}, \hat{\boldsymbol{\beta}}^{-}\right)$. Details for solving (1) can be seen in Algorithm 1. Isotonic regression can be computed in $O(N)$ operations (Grotzinger \& Witzgall 1984) and hence the ordered lasso algorithm can be applied to large datasets.

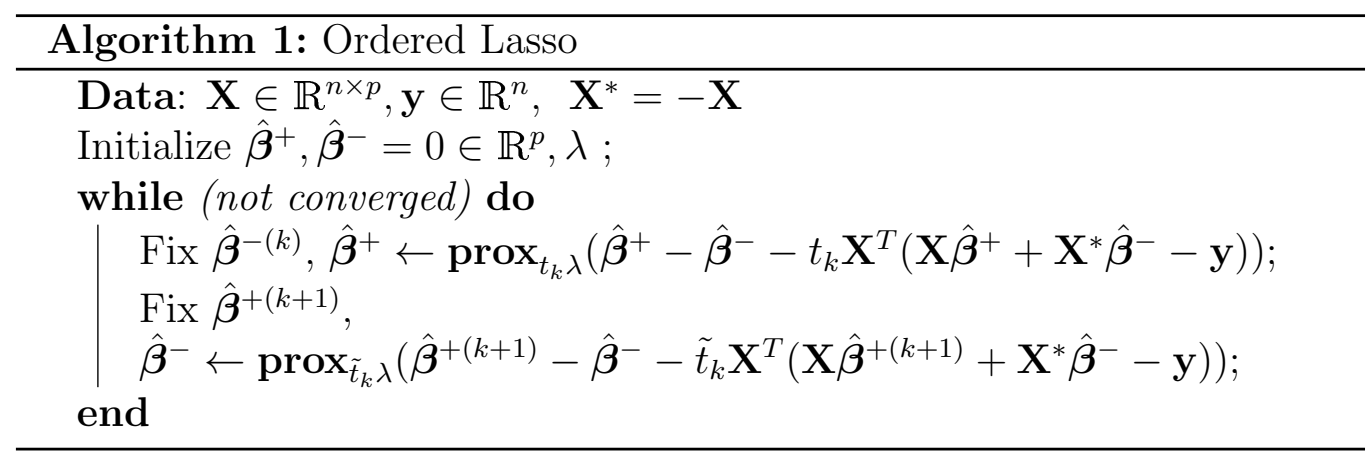

The ordered lasso can be easily adapted to the elastic net (Zou \& Hastie 2005 ) and the adaptive lasso (Zou 2006) by some simple modifications to the proximal operator in Equation (6).

\subsection{Comparison between the ordered lasso and the lasso}

Figure 1 shows a comparison between the ordered lasso and the standard lasso. The data was generated from a true monotone sequence plus Gaussian noise. The black profiles show the true coefficients, while the colored profiles are the estimated coefficients for different values of $\lambda$, from largest (at bottom) to smallest (at top). The corresponding plot for the lasso is shown in the bottom panel. The ordered lasso - exploiting the monotonicity - does a much better job of recovering the true coefficients than the lasso, as seen by the fluctuations of the estimated coefficients in the tails of the lasso plot. 


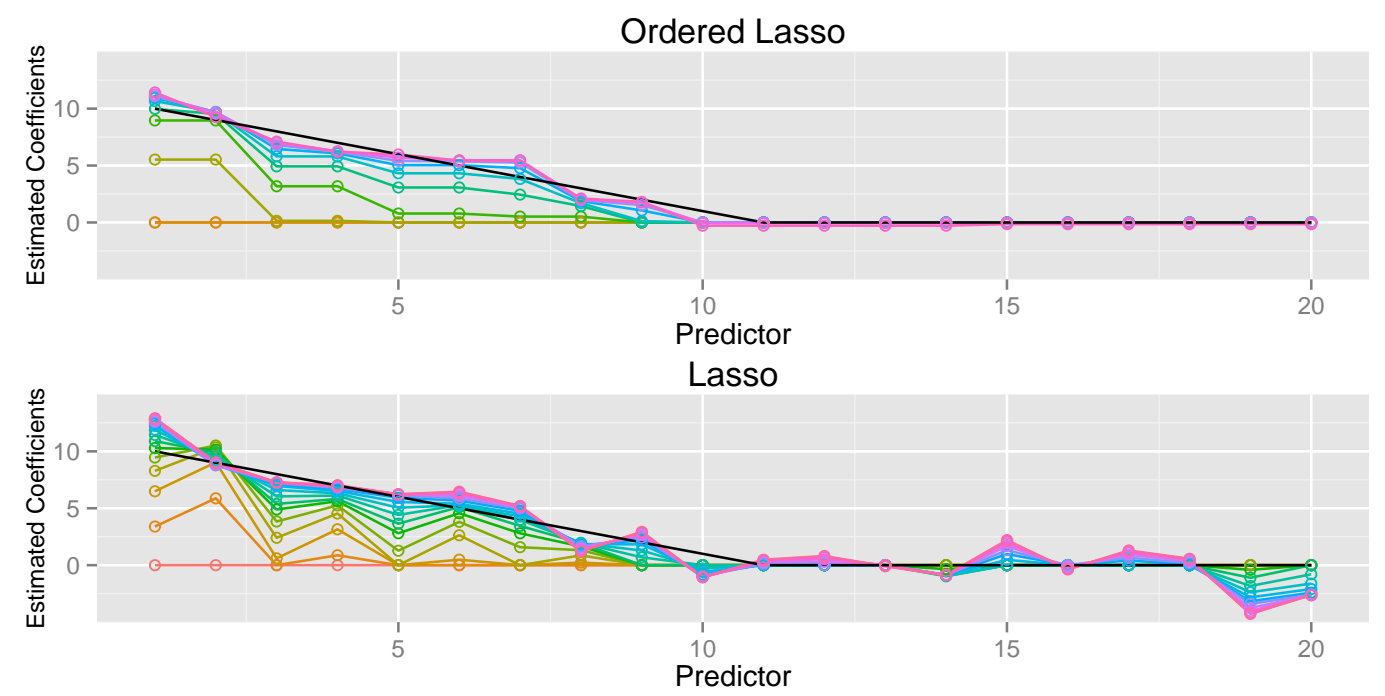

Figure 1: Example of the ordered lasso compared to the standard lasso: the data was generated from a true monotone sequence of coefficients plus Gaussian noise: $y_{i}=\sum_{j=1}^{p} x_{i j} \beta_{j}+\sigma \cdot Z_{i}$, with $x_{i j} \sim N(0,1), \boldsymbol{\beta}=$ $(10,9, \ldots, 2,1,0,0, \ldots 0), \sigma=7$. There were 20 predictors and 30 observations. The black profiles show the true coefficients and the colored profiles are the estimated coefficients for different values of $\lambda$ from the largest(at bottom) to the smallest(at top).

\subsection{Relaxation of the monotonicity requirement}

As a generalization of our approach, we can relax problem (1) as follows

$$
\begin{array}{r}
\operatorname{minimize}\left\{\frac{1}{2} \sum_{i=1}^{N}\left(y_{i}-\beta_{0}-\sum_{j=1}^{p} x_{i j} \beta_{j}\right)^{2}+\lambda \sum_{j=1}^{p}\left(\beta_{j}^{+}+\beta_{j}^{-}\right)\right. \\
\left.+\theta_{1} \sum_{j=1}^{p-1}\left(\beta_{j}^{+}-\beta_{j+1}^{+}\right)_{+}+\theta_{2} \sum_{j=1}^{p-1}\left(\beta_{j}^{-}-\beta_{j+1}^{-}\right)_{+}\right\},
\end{array}
$$

subject to $\beta_{j}^{+}, \beta_{j}^{-} \geq 0, \forall j$. As $\theta_{1}, \theta_{2} \rightarrow \infty$, the last two penalty terms force monotonicity and this is equivalent to (1). However, for intermediate positive values of $\theta_{1}, \theta_{2}$, these penalties encourage near-monotonicity. This idea was proposed in Tibshirani, Hoefling \& Tibshirani (2011) for data sequences, generalizing the isotonic regression problem. The authors derive an efficient algorithm NearIso which is a generalization of the well-known PAVA procedure mentioned above. Operationally, this creates no extra complication in 
our framework: we simply use NearIso in place of PAVA in the generalized gradient algorithm described in Section 2.2.

\section{Sparse time-lagged regression}

In this section we apply the ordered lasso to the time-lagged regression problem. There are two problems we consider. The first one is the static outcome problem, where we observe outcome at a fixed time $t$ and predictors at a series of time points, and the outcome at time $t$ is predicted from the predictors at previous time points. We also consider the rolling prediction problem where we observe both outcome and predictors at a series of time points and the outcome is predicted at each time point from the predictors at previous time points. Again, we assume that the predictors and outcome are centered so that the intercept has the solution $\hat{\beta}_{0}=0$. Henceforth we will continue to omit the intercept.

\subsection{Static prediction from time-lagged features}

Here we consider the problem of predicting an outcome at a fixed time point from a set of time-lagged predictors. We assume that our data has the form $\left\{y_{i}, x_{i 11}, \ldots x_{i K 1}, x_{i 12}, \ldots x_{i K 2}, \ldots x_{i 1 p}, \ldots, x_{i K p}\right\}$, for $i=1,2, \ldots, N$ and $N$ being the number of observations. The value $x_{i k j}$ is the measurement of predictor $j$ of observation $i$, at time-lag $k$ from the current time $t$. In other words, we predict the outcome at time $t$ from $p$ predictors, each measured at $K$ time points preceding the current time $t$. Our model has the form

$$
y_{i}=\beta_{0}+\sum_{j=1}^{p} \sum_{k=1}^{K} x_{i k j} \beta_{k j}+\epsilon_{i},
$$

with $\mathrm{E}\left(\epsilon_{i}\right)=0$ and $\operatorname{Var}\left(\epsilon_{i}\right)=\sigma^{2}$. We write each $\beta_{k j}=\beta_{k j}^{+}-\beta_{k j}^{-}$and solve

$$
\operatorname{minimize}\left\{\frac{1}{2} \sum_{i=1}^{N}\left(y_{i}-\hat{y}_{i}\right)^{2}+\lambda \sum_{j=1}^{p} \sum_{k=1}^{K}\left(\beta_{k j}^{+}+\beta_{k j}^{-}\right)\right\},
$$

subject to $\beta_{1 j}^{+} \geq \beta_{2 j}^{+} \geq \ldots \geq \beta_{K j}^{+} \geq 0$ and $\beta_{1 j}^{-} \geq \beta_{2 j}^{-} \geq \ldots \geq \beta_{K j}^{-} \geq 0, \forall j$. This model makes the plausible assumption that each predictor has an effect up to $K$ time units away from the current time $t$, and this effect is monotone non-increasing as we move farther back in time. 
In order to solve $(8)$, we first write each $\beta_{k j}^{ \pm}$in the following form,

$$
\{\underbrace{\beta_{11}^{ \pm}, \beta_{21}^{ \pm}, \cdots, \beta_{K 1}^{ \pm}}_{\text {block } 1}|\underbrace{\beta_{12}^{ \pm}, \beta_{22}^{ \pm}, \cdots, \beta_{K 2}^{ \pm}}_{\text {block } 2}| \cdots \mid \underbrace{\beta_{1 p}^{ \pm}, \beta_{2 p}^{ \pm}, \cdots, \beta_{K p}^{ \pm}}_{\text {block p }}\} \text {. }
$$

This is a blockwise coordinate descent procedure, with one block for each predictor. For example, at step $j$, we compute the update for block $j$ while holding the rest of the blocks constant. We augment each predictor $x_{i k j}$ by $x_{i k j}^{*}=-x_{i k j}$. With a sufficiently large time-lag $K$, the procedure automatically chooses an appropriate number of non-zero coefficients for each predictor, and zeros out the rest in each block because of the order constraint on each predictor. Details can be seen in Algorithm 2.

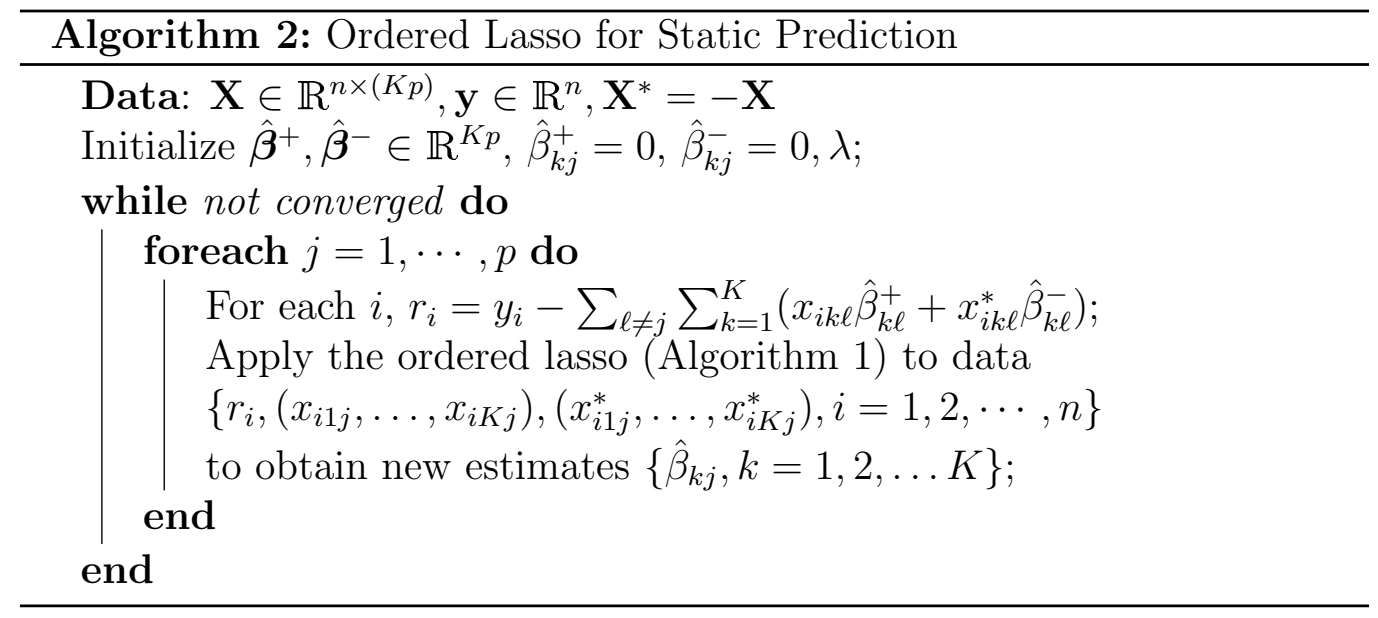

\subsection{Rolling prediction from time-lagged features}

Here we assume that our data has the form $\left\{y_{t}, x_{t 1}, \ldots, x_{t p}\right\}$, for $t=1,2, \ldots, N$. In detail, we have a time series for which we observe the outcome and the values of each predictor at $N$ different time points. We consider a time-lagged regression model with a maximum lag of $K$ time points

$$
y_{t}=\beta_{0}+\sum_{j=1}^{p} \sum_{k=1}^{K} x_{t-k, j} \beta_{k j}+\epsilon_{t}
$$


with $\mathrm{E}\left(\epsilon_{t}\right)=0$ and $\operatorname{Var}\left(\epsilon_{t}\right)=\sigma^{2}$. We write each $\beta_{k j}=\beta_{k j}^{+}-\beta_{k j}^{-}$and propose the following problem

$$
\operatorname{minimize}\left\{\frac{1}{2} \sum_{t=1}^{N}\left(y_{t}-\hat{y}_{t}\right)^{2}+\lambda \sum_{j=1}^{p} \sum_{k=1}^{K}\left(\beta_{k j}^{+}+\beta_{k j}^{-}\right)\right\},
$$

subject to $\beta_{1 j}^{+} \geq \beta_{2 j}^{+} \geq \ldots \geq \beta_{K j}^{+} \geq 0$ and $\beta_{1 j}^{-} \geq \beta_{2 j}^{-} \geq \ldots \geq \beta_{K j}^{-} \geq 0, \forall j$. To solve this problem, we convert the problem into the form of Section 3.1. We build a larger feature matrix $\mathbf{Z}$ of size $N \times(K p)$, with $K$ columns for each predictor. In detail, each row has the form

$$
\left\{x_{t-1,1}, x_{t-2,1}, \ldots, x_{t-K, 1}\left|x_{t-1,2}, x_{t-2,2}, \ldots, x_{t-K, 2}\right| \ldots \mid x_{t-1, p}, x_{t-2, p}, \ldots, x_{t-K, p}\right\} .
$$

Each block corresponds to a predictor lagged for $1,2, \ldots, K$ time units. The matrix $\mathbf{Z}$ has $N$ such rows, corresponding to time points $t-1, t-2, \ldots t-K$. Again, we augment each predictor $x_{t-k, j}$ with $x_{t-k, j}^{*}=-x_{t-k, j}$ and choose a sufficiently large time-lag $K$, and let the procedure to zero out extra coefficients for each predictor. We can solve (9) using block coordinate descent as in the previous subsection. Details are shown in Algorithm 3.

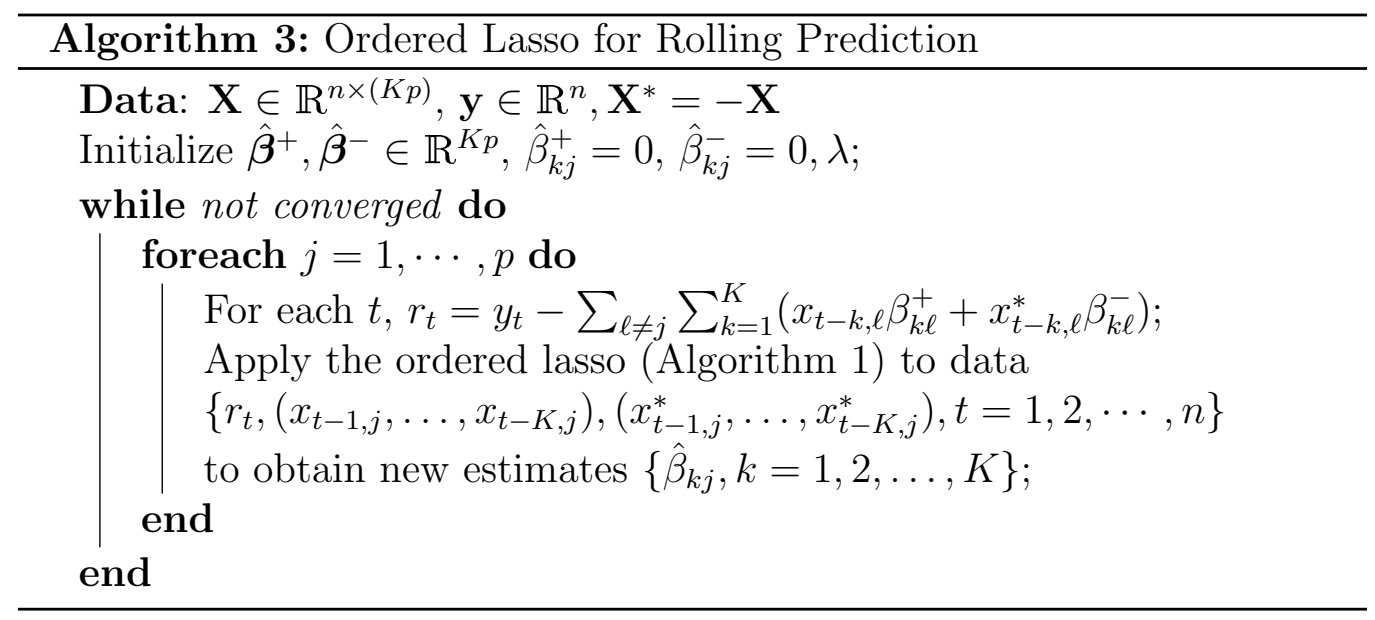

\subsection{Simulated example}

Figure 2 shows an example of the ordered lasso procedure applied to a rolling time-lagged regression. The simulated data consists of four predictors with a 
maximum lag of 5 time points and 111 observations. The true coefficients for each of the four predictors were $(7,5,4,2,0),(5,3,0,0,0),(3,0,0,0,0)$ and $(0,0,0,0,0)$. The features were generated as i.i.d. $N(0,1)$ with Gaussian noise of a standard deviation equal to 7 . The figure shows the true coefficients (black), and estimated coefficients of the ordered lasso (blue) and the standard lasso (orange) from 20 simulations. For each method, the coefficient estimates with the smallest mean squared error (MSE) in each realization are plotted. We see that the ordered lasso does a better job of recovering the true coefficients. The average mean squared errors for the ordered lasso and the lasso were $4.08(.41)$ and $6.11(.54)$, respectively.
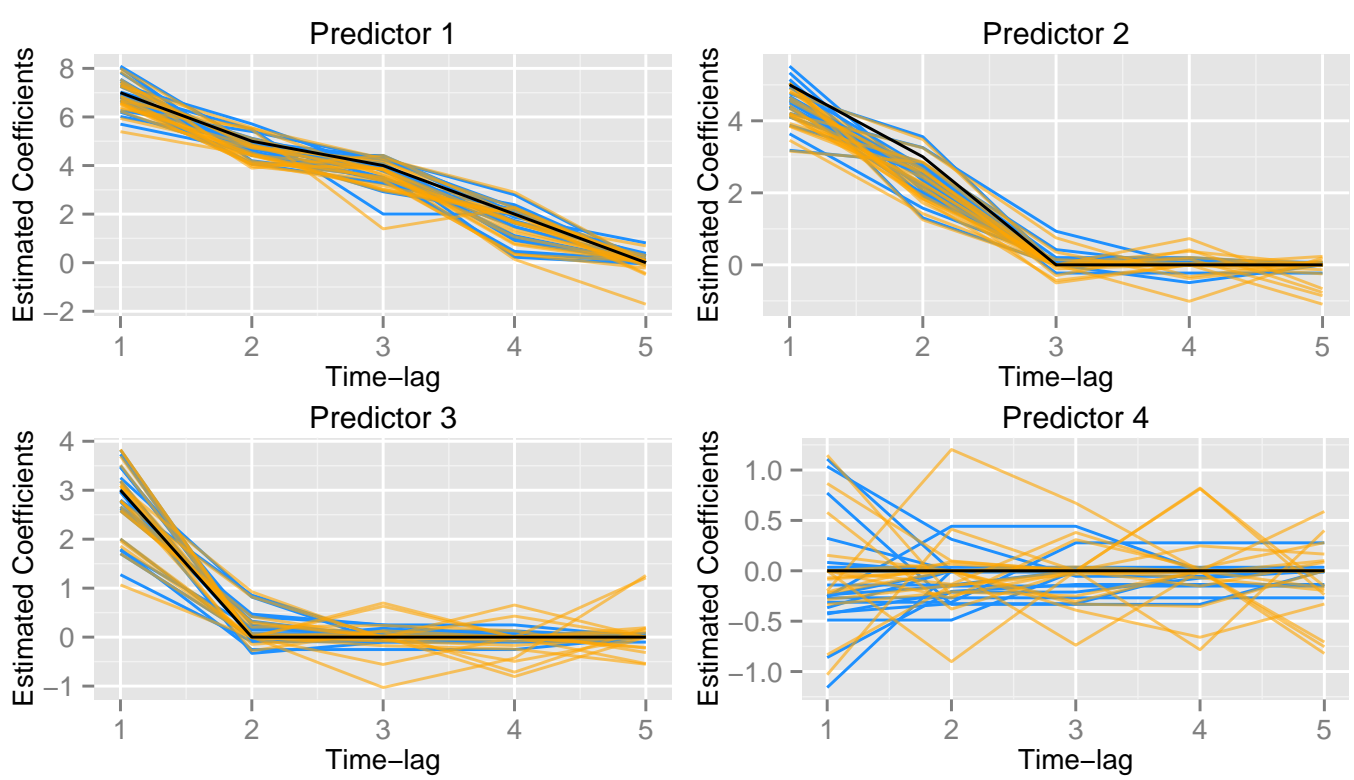

Figure 2: True coefficients (black), coefficient estimates the ordered lasso (blue) and the standard lasso (orange) from 20 simulations.

Figure 3 shows a larger example with a maximum lag of 20 time points. The features were generated as i.i.d. $N(0,1)$ with Gaussian noise of a standard deviation equal to 7 . Let $f(a, b, L)$ denote the equally spaced sequence from $a$ to $b$ of length $L$. The true coefficients for each of the four predictors were $\{f(5,1,20)\},\{f(5,1,10), f(0,0,10)\},\{f(5,1,5), f(0,0,15)\}$ and $\{f(0,0,20)\}$. The left panel of the figure shows the mean squared error of the standard lasso and the ordered lasso, over 30 simulations. The value 

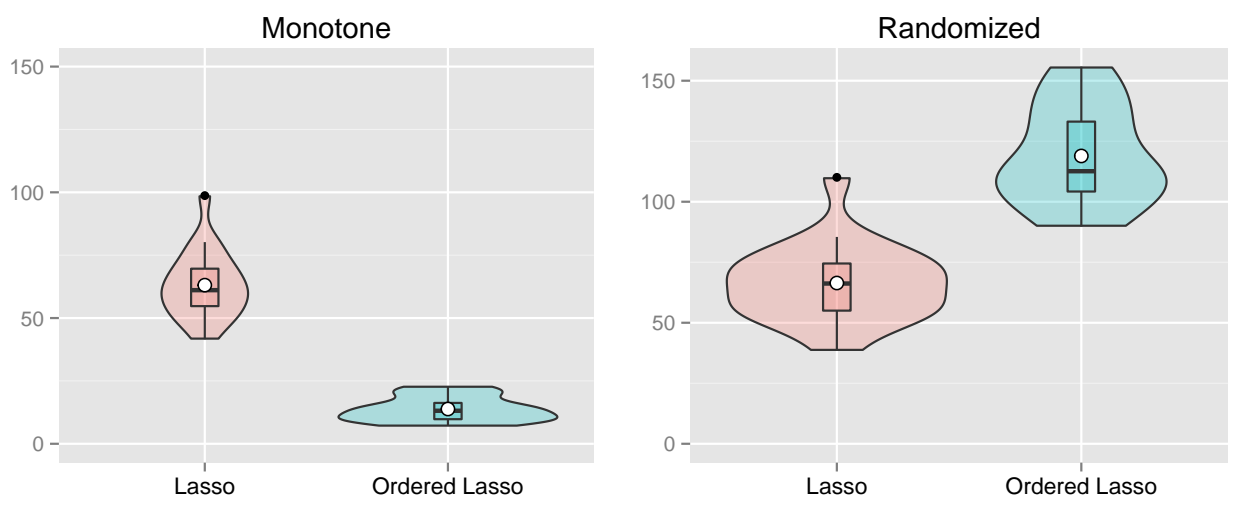

Figure 3: The lasso and the ordered lasso, applied to time-lagged features. Shown is the mean squared error over 30 simulations using the minimizing value of $\lambda$ for each realization. In the left panel, the true coefficients are monotone; in the right, they have been scrambled so that monotonicity does not hold.

of $\lambda$ giving the minimum MSE was chosen in each realization. In the right panel we have randomly permuted the true predictor coefficients for each realization, thereby causing the monotonicity to be violated (on average), but keeping the same signal-to-noise ratio. Not surprisingly, the ordered lasso does better when the true coefficients are monotone, while the reverse is true for the lasso. However, we also see that in an absolute sense one can achieve a much lower MSE in the monotone setting of the left panel.

\subsection{Performance on Los Angeles ozone data}

These data are available at http://statweb.stanford.edu/ tibs/ElemStatLearn/ data.html. They represent the level of atmospheric ozone concentration from eight daily meteorological measurements made in the Los Angeles basin for 330 days in 1976. The response variable is the log of the daily maximum of the hourly-averaged ozone concentrations in Upland, California. We divided the data into training and validation sets of approximately the same size, and considered models with a maximum time-lag of 20 days.

Figure 4 shows the prediction error curves over the validation set, for the "cross-sectional" lasso (predicting from measurements on the same day), the lasso (predicting from measurements on the same day and the previous 19 


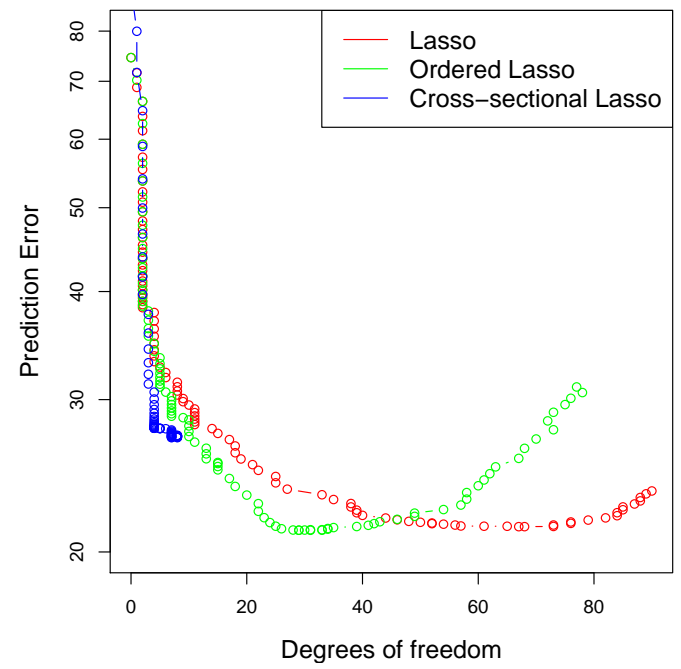

Figure 4: Ozone data: prediction error curves. The cross-sectional lasso (blue) predicts from measurements on the same day, the lasso(red) predicts from measurements on the same day and previous 19 days, and the ordered lasso (green) adds the monotonicity constraint to the lasso.

days), and the ordered lasso, which adds the monotonicity constraint to the lasso. We see that the ordered lasso and the lasso applied to time-lagged features achieve lower errors than the "cross-sectional" lasso. In addition, the ordered lasso achieves the minimum with fewer degrees of freedom(as defined in Section 4).

Figure 5 shows the estimated coefficients from the ordered lasso (top) and the lasso (bottom). The ordered lasso yields simpler and more interpretable solutions. For each predictor, the ordered lasso also determines the most suitable estimate of the time-lag interval, beyond which the estimated coefficients are zero. For example, the estimated coefficients of the predictor "wind" are zero beyond a time-lag of 14 days from the current time $t$, whereas the estimated coefficients "humidity" are zero beyond a time-lag of 7 days from the current time $t$. 


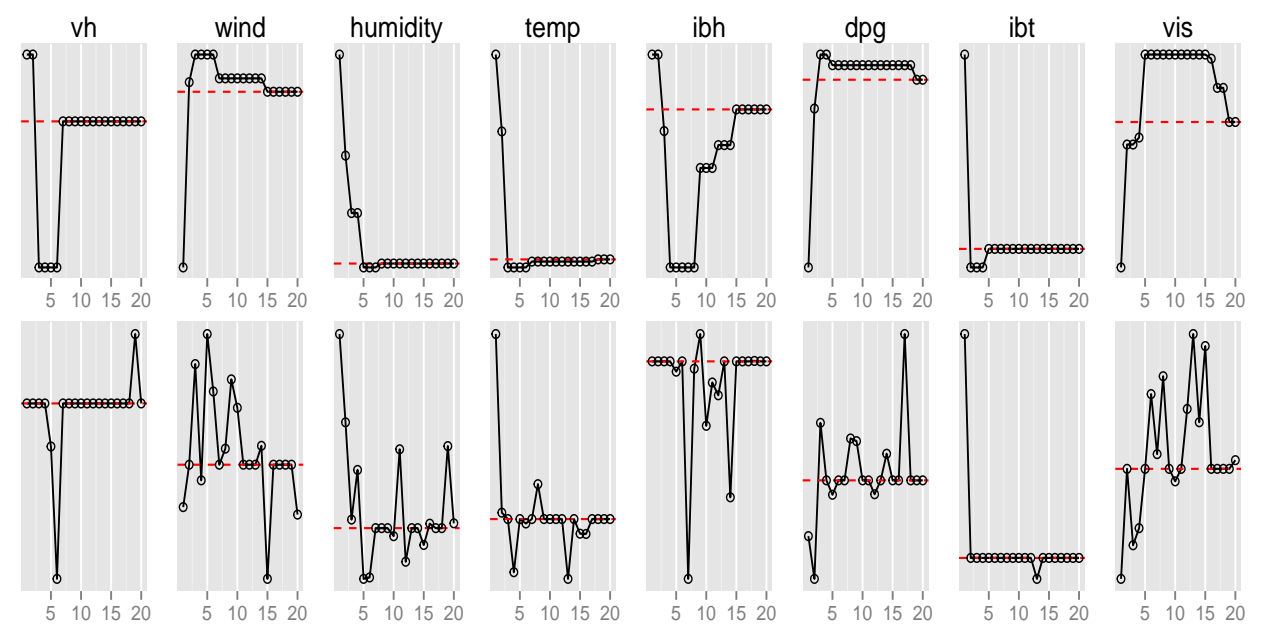

Figure 5: Ozone data: estimated coefficients from the ordered lasso (top) and the lasso (bottom) versus time-lag. For reference, a dashed red horizontal line is drawn at zero.

\subsection{Auto-regressive time series applied to sunspot data and simulated data}

In an auto-regressive time series model, one predicts each value $y_{t}$ from the values $y_{t-1}, y_{t-2} \ldots y_{t-k}$ for some maximum lag, or "order" $k$. This fits into the time-lagged regression framework, where the regressors are simply the time series itself at previous time points. Our proposal for monotone constraints in the AR model seems to be novel. Nardi \& Rinaldo (2011) studied the application of the standard lasso to the AR model and derived its asymptotic properties. Schmidt \& Makalic (2013) suggested a Bayesian approach to the lasso based on the partial autocorrelation representation of AR models. In the following example, we compare coefficient estimates and order estimates among the ordered lasso, the lasso, and the standard AR fit.

The data for this example is available in the $\mathrm{R}$ package as sunspot.year. The data contains 289 measurements and they represent yearly numbers of sunspots from 1700 to 1988. Figure 6 shows the results of the auto-regressive model fit to the yearly sunspot data. We separated the series into training and validation series of about equal size. The standard AR fit (right panel) chose an order of 9 using least squares and the Akaike information criterion (AIC). The ordered lasso (with $\lambda$ chosen by two-fold cross validation) suggests 


\begin{tabular}{l|rrrrrrrrrr}
\hline Method Est. lag & 1 & 2 & 3 & 4 & 5 & 6 & 7 & 8 & 9 & 10 \\
\hline AR/AIC & 0 & 0 & 69 & 14 & 6 & 2 & 3 & 3 & 0 & 3 \\
\hline Ordered Lasso & 0 & 0 & 66 & 14 & 3 & 5 & 4 & 1 & 3 & 4 \\
\hline
\end{tabular}

Table 1: Estimates of AR lag from the ordered lasso, and AR model using least squares and $A I C$ from 100 simulations. The data was generated as $y_{i}=$ $\sum_{k=1}^{3} y_{i-k} \beta_{k}+\sigma \cdot Z_{i}$ where $\sigma=4, y_{i}, Z_{i} \sim N(0,1)$, and $\boldsymbol{\beta}=\{0.35,0.25,0.25\}$. Each entry represents the number of times that a specific lag was estimated in 100 simulations.

an order of 10 (out of a maximum of 20) and gives a well-behaved sequence of coefficients. The regular lasso (middle panel) — with no monotonicity constraints - gives a less clear picture. All three estimates had about the same error on the validation set.
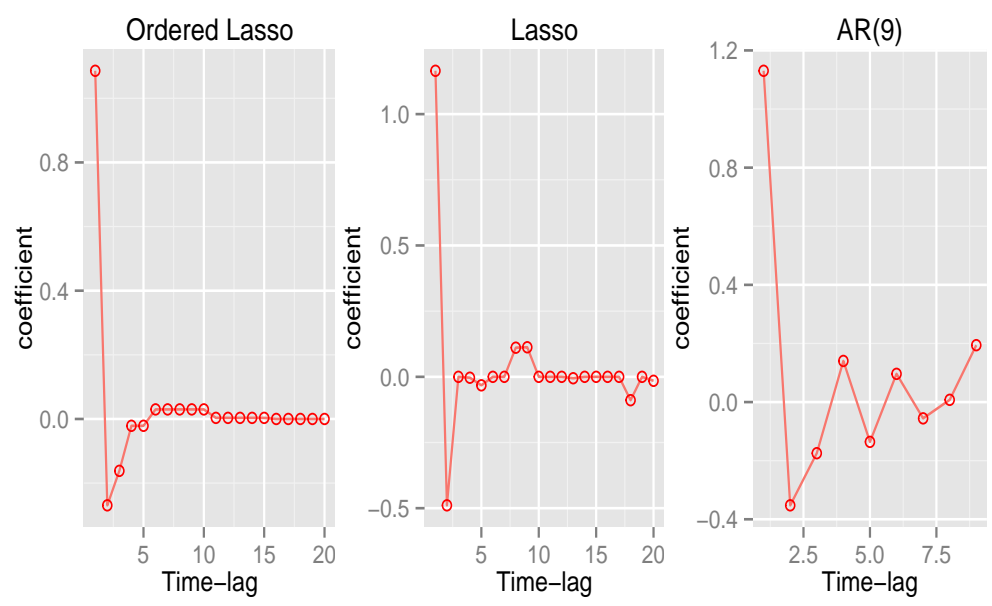

Figure 6: Sunspot data: estimated coefficients of the ordered lasso, the lasso and the standard $A R$ fit.

Table 1 shows the results of an experiment comparing the ordered lasso to the standard AR fitting using AIC from 100 simulations. The goal was to estimate the lag of the time series (number of non-zero coefficients), as in the previous figure. The true series was of length 1000, with an actual lag of 3 , and the maximum lag considered was 10 . The data was divided into training and validation series of approximately the same size. The ordered 
lasso used the second half of the series to estimate the best value of $\lambda$ and estimate the order of the series. The results show that the ordered lasso has similar performance to AR/AIC for this task.

\section{Degrees of freedom}

Given a fit vector $\hat{\mathbf{y}}$ for estimation from a vector $\mathbf{y} \sim N\left(\boldsymbol{\mu}, \mathbf{I} \cdot \sigma^{2}\right)$, the degrees of freedom of the fit can be defined as

$$
\operatorname{df}(\hat{\mathbf{y}})=\frac{1}{\sigma^{2}} \sum_{i=1}^{N} \operatorname{Cov}\left(y_{i}, \hat{y}_{i}\right)
$$

(Efron 1986). This applies even if $\mathbf{y}$ is an adaptively chosen estimate. Zou, Hastie \& Tibshirani (2007) show that for the lasso, the number of nonzero "plateaus" (coefficients) in the solution is an unbiased estimate of the degrees of freedom. Tibshirani \& Taylor (2011) give analogous estimates for generalized penalties. For near-isotonic regression described in Section 2.4. letting $\hat{k}$ denote the number of nonzero "plateaus" in the solution, Tibshirani et al. (2011) show that

$$
\mathrm{E}(\hat{k})=\operatorname{df}(\hat{\mathbf{y}})
$$

For the ordered lasso, this can be applied directly in the orthogonal design case to yield (11). For the general $\mathbf{X}$, we conjecture that the same result holds, and can be established by studying the properties of projection onto the convex constraint set (as detailed in Tibshirani \& Taylor (2011)).

\section{$5 \quad$ Logistic regression model}

Here we show how to generalize the ordered lasso to logistic regression. Assume that we observe $\left(\mathbf{x}_{i}, y_{i}\right), i=1,2, \ldots, N$ with $\mathbf{x}_{i}=\left(x_{i 1}, \ldots, x_{i p}\right)$ and $y_{i}=0$ or 1 . The log-likelihood function is

$$
l(\boldsymbol{\beta})=\sum_{i=1}^{N}\left(y_{i}\left(\beta_{0}+\mathbf{x}_{i}^{T} \boldsymbol{\beta}\right)-\log \left(1+e^{\beta_{0}+\mathbf{x}_{i}^{T} \boldsymbol{\beta}}\right)\right) .
$$


With the ordered lasso, we write each $\beta_{j}=\beta_{j}^{+}-\beta_{j}^{-}$with $\beta_{j}^{+}, \beta_{j}^{-} \geq 0$, and solve

$$
\operatorname{maximize}\left\{l\left(\boldsymbol{\beta}^{+}-\boldsymbol{\beta}^{-}\right)-\lambda\left(\sum_{j=1}^{p}\left(\beta_{j}^{+}+\beta_{j}^{-}\right)\right\},\right.
$$

subject to $\beta_{1}^{+} \geq \cdots \geq \beta_{p}^{+} \geq 0$ and $\beta_{1}^{-} \geq \cdots \geq \beta_{p}^{-} \geq 0$. We write our data in matrix form and use the iteratively reweighted least squares method (IRLS) to solve 12 , i.e., at each iteration, we solve

$$
\begin{gathered}
\operatorname{minimize}\left\{\frac{1}{2}\left(\mathbf{z}-\beta_{0}-\mathbf{X}\left(\boldsymbol{\beta}^{+}-\boldsymbol{\beta}^{-}\right)\right)^{T} \mathbf{W}\left(\mathbf{z}-\beta_{0}-\mathbf{X}\left(\boldsymbol{\beta}^{+}-\boldsymbol{\beta}^{-}\right)\right)\right. \\
\left.+\lambda \sum_{i=1}^{p}\left(\beta_{i}^{+}+\beta_{i}^{-}\right)\right\}
\end{gathered}
$$

subject to $\beta_{1}^{+} \geq \beta_{2}^{+} \geq \cdots \geq \beta_{p}^{+} \geq 0$ and $\beta_{1}^{-} \geq \beta_{2}^{-} \geq \cdots \geq \beta_{p}^{-} \geq 0$, where $\mathbf{z}=\beta_{0}^{\text {old }}+\mathbf{X}\left(\boldsymbol{\beta}_{\text {old }}^{+}-\boldsymbol{\beta}_{\text {old }}^{-}\right)+\mathbf{W}^{-1}(\mathbf{y}-\mathbf{p})$, $\mathbf{p}$ is a vector with $\mathbf{p}_{i}=$ $\left.\frac{\exp \left(\beta_{0}^{\text {old }}+\mathbf{x}_{i}^{T}\left(\boldsymbol{\beta}_{\text {old }}^{+}-\boldsymbol{\beta}_{\text {old }}^{-}\right)\right)}{1+\exp \left(\beta_{0}^{\text {old }}+\mathbf{x}_{i}^{T}\left(\boldsymbol{\beta}_{\text {old }}^{+}-\boldsymbol{\beta}_{\text {old }}^{-}\right)\right.}\right)$, and $\mathbf{W}$ is a diagonal matrix with $\mathbf{W}_{i i}=\mathbf{p}_{i}\left(1-\mathbf{p}_{i}\right)$. We apply the ordered lasso (Algorithm 1) to solve (13) with modified updates:

$$
\begin{aligned}
& \beta_{0} \leftarrow \beta_{0}-\gamma \mathbf{1}^{T} \mathbf{W}\left(\beta_{0}+\mathbf{X} \boldsymbol{\beta}-\mathbf{z}\right), \\
& \boldsymbol{\beta} \leftarrow \operatorname{prox}_{\gamma \lambda}\left(\boldsymbol{\beta}-\gamma \mathbf{X}^{T} \mathbf{W}\left(\beta_{0}+\mathbf{X} \boldsymbol{\beta}-\mathbf{z}\right)\right) .
\end{aligned}
$$

Applying the ordered lasso to the logistic regression model with time-lagged features, we approximate the log-likelihood function as in (13) and use Algorithm 2 or Algorithm 3 to solve the weighted least squares minimization subproblem. Similar extensions can be made to other generalized linear models.

\section{Discussion}

In this paper, we have proposed an order-constrained version of the lasso. This procedure has natural applications to the static and rolling prediction problems, based on time-lagged variables. It can be applied to any dynamic prediction problem, including financial time series and prediction of dynamic patient outcomes based on clinical measurements. For the future work, we could generalize our framework to higher dimensional notions of monotonicity, which could be useful for spatial data. An R package that implements the algorithms will be made available on the CRAN website. 


\section{Acknowledgement}

We thank Stephen Boyd for his helpful suggestions.

\section{References}

Barlow, R. E., Bartholomew, D., Bremner, J. M. \& Brunk, H. D. (1972), Statistical inference under order restrictions; the theory and application of isotonic regression, Wiley, New York.

Bien, J., Taylor, J. \& Tibshirani, R. (2013), 'A lasso for hierarchical interactions', Annals of Statistics 42(3), 1111-1141.

de Leeuw, J., Hornik, K. \& Mair, P. (2009), 'Isotone optimization in R: Pool-adjacent-violators (PAVA) and active set methods', Journal of Statistical Software 32(5), 1-24.

Efron, B. (1986), 'How biased is the apparent error rate of a prediction rule?', Journal of the American Statistical Association 81, 461-70.

Grotzinger, S. J. \& Witzgall, C. (1984), 'Projections onto simplices', Applied Mathematics and Optimization 12(1), 247-270.

Nardi, Y. \& Rinaldo, A. (2011), 'Autoregressive process modeling via the lasso procedure', Journal of Multivariate Analysis 102(3), 528 - 549.

Schmidt, D. F. \& Makalic, E. (2013), 'Estimation of stationary autoregressive models with the bayesian lasso', Journal of Time Series Analysis pp. $\mathrm{n} / \mathrm{a}-\mathrm{n} / \mathrm{a}$.

Tibshirani, R. (1996), 'Regression shrinkage and selection via the lasso', Journal of the Royal Statistical Society, Series B 58, 267-288.

Tibshirani, R., Hoefling, H. \& Tibshirani, R. (2011), 'Nearly-isotonic regression', Technometrics 53(1), 54-61.

Tibshirani, R. \& Taylor, J. (2011), 'On the degrees of freedom of the lasso', Annals of Statistics (40), 1198-1232.

Zou, H. (2006), 'The adaptive lasso and its oracle properties', Journal of the American Statistical Association 101, 1418-1429. 
Zou, H. \& Hastie, T. (2005), 'Regularization and variable selection via the elastic net', Journal of the Royal Statistical Society Series B 67(2), 301320.

Zou, H., Hastie, T. \& Tibshirani, R. (2007), 'On the "degrees of freedom" of the lasso', Annals of Statistics 35(5), 2173-2192. 